فرهاد غفورى كسبى'، قدرت رحيمى ميانجى'، محمود هنرور 'و اردشير نجاتى جوارمى'

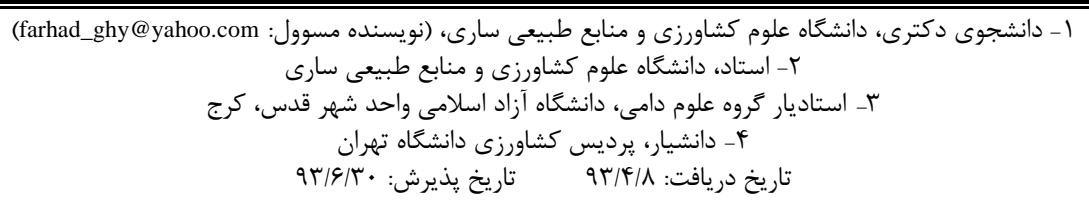

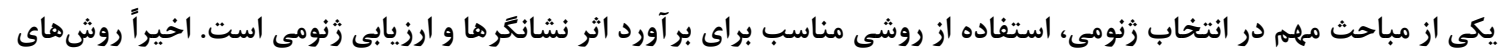

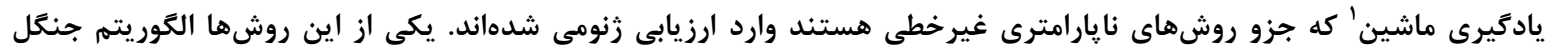

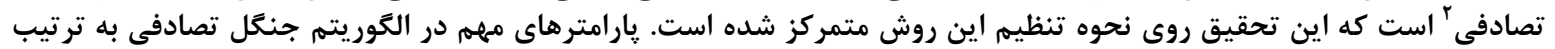

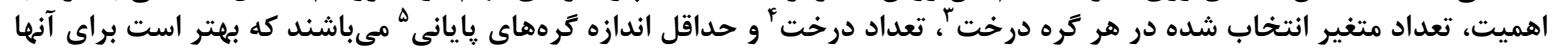

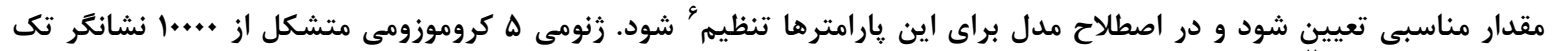

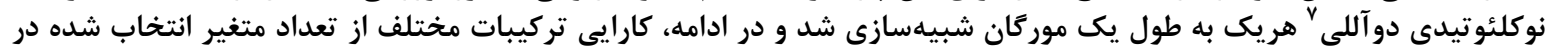

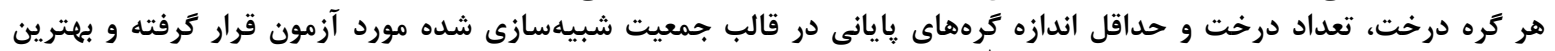

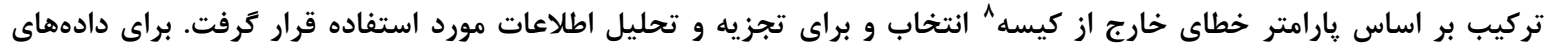

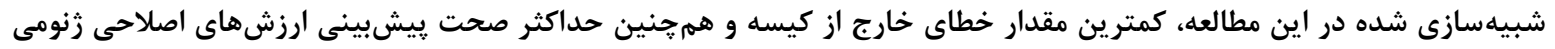

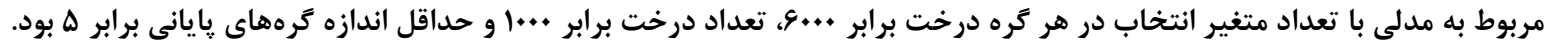

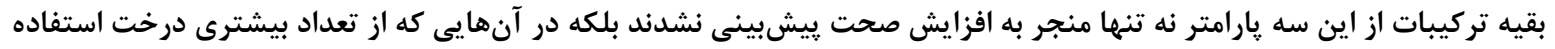

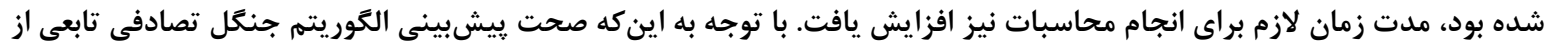

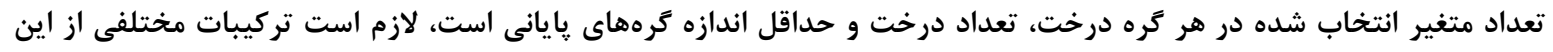

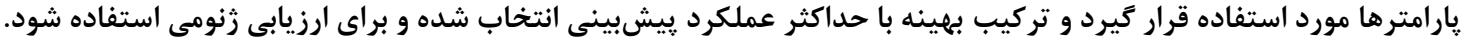

$$
\text { وازههاى كليدى: ارزيابى زنومى، جنعل تصادفى، درخت، نشانكَر تك نوكلئوتيدى، ارزشهاى اصلاحى }
$$

مربوط به ماندكارى و صفاتى كه اندازمخيرى آنها مشكل

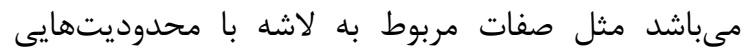

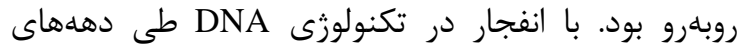

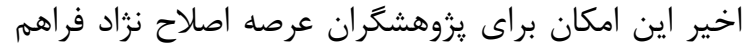

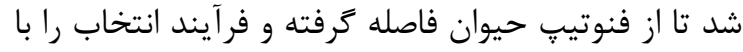

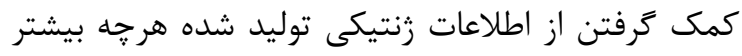

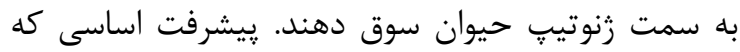

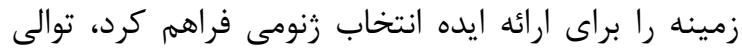

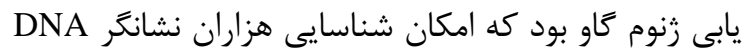

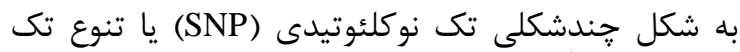

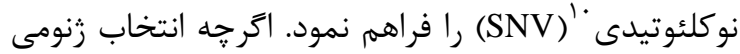

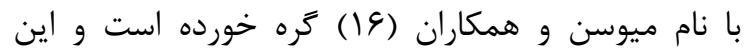

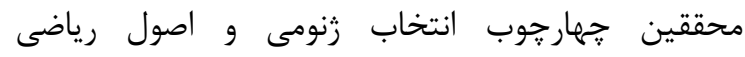

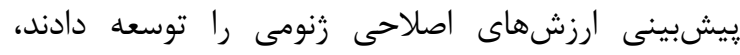

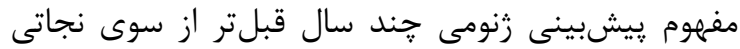

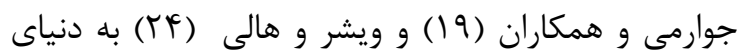
1- Machine Learning

7- Ntree 10- Single Nucleotide Variation

\section{2- Random}

5- Nodesize

8- Out Of Bag Error (OOB Error)
مقدمه

در روشهاى رايج اصلاح نزاد دام، معمولاً اصلاحكران

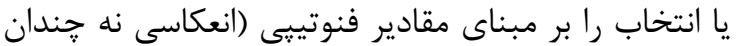

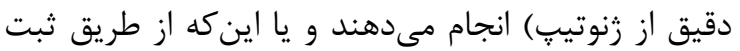

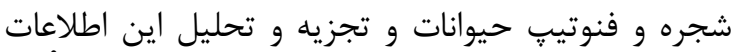

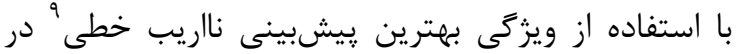

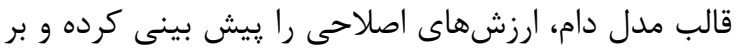

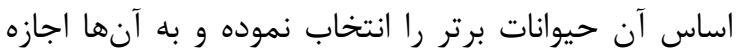

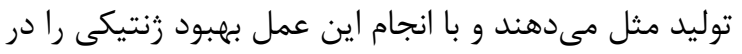

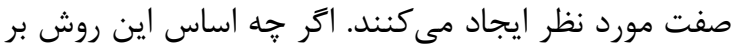

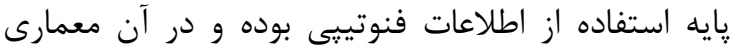

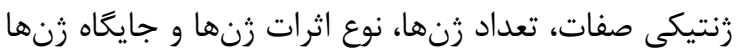

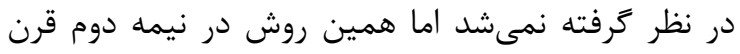

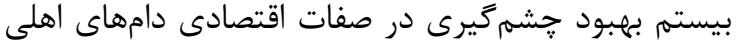

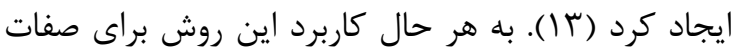

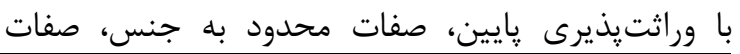
3- Mtry 6- Tune 9- Best Linear Unbiased Prediction 
ابعاد بالا و يا اطلاعات توالىيابى زنومى با حجم بسيار بالا،

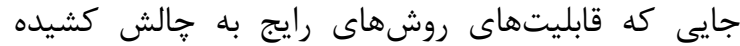

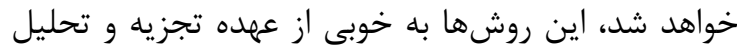

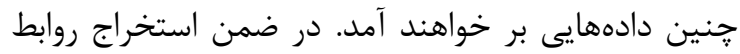

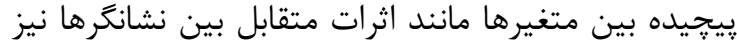

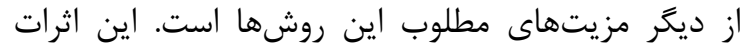

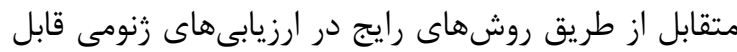

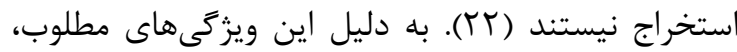

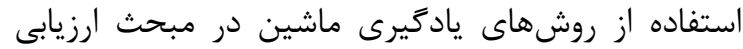

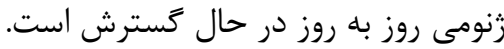

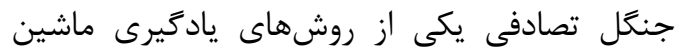

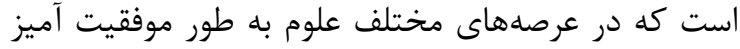

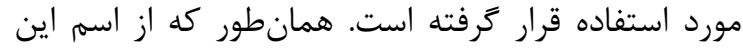

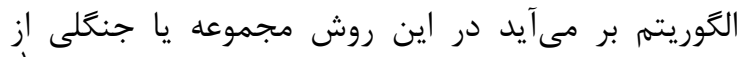

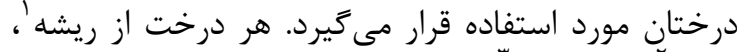

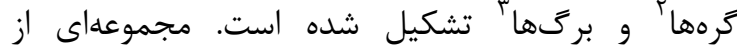
مثالهاى آموزشى

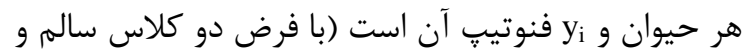

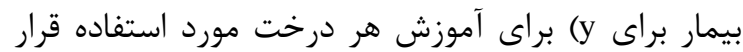

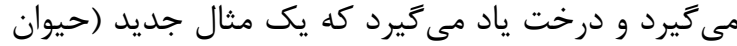

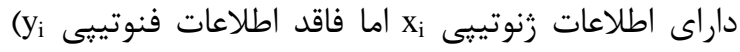

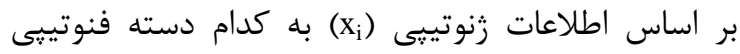

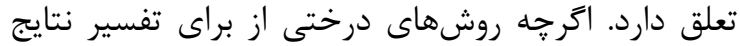

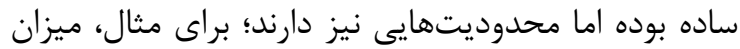

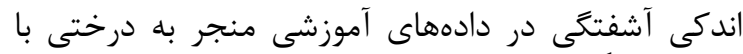

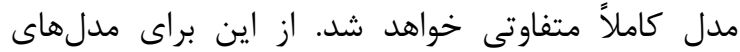

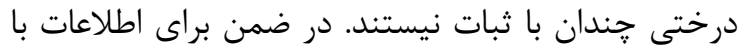

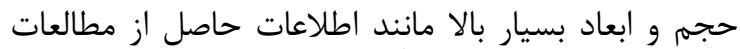

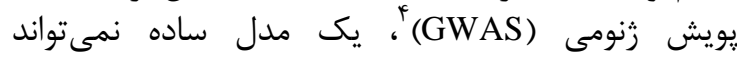

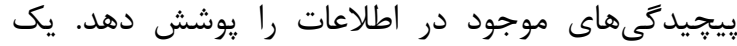

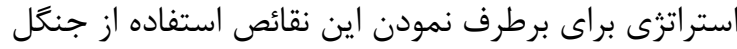

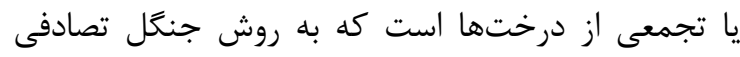

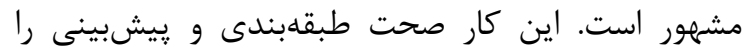

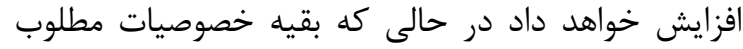

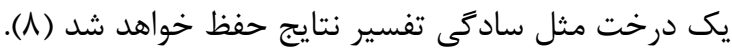

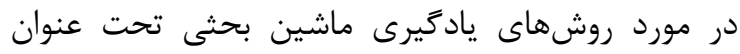

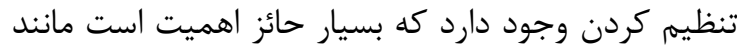

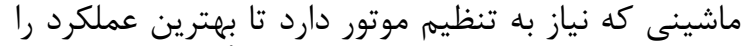

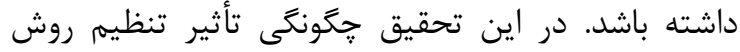

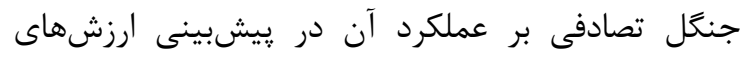
اصلاحى زنومى مورد بررسى قرار كرفتئه است.
اصلاح نزاد دام وارد شده بود. در دسترس بودن انبوه

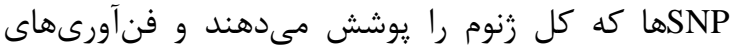

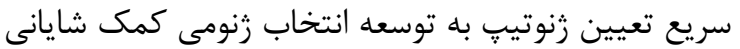

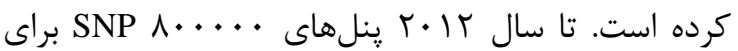

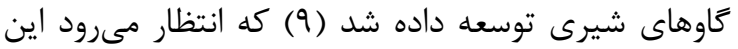

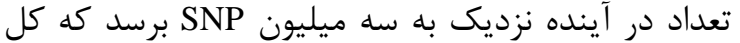

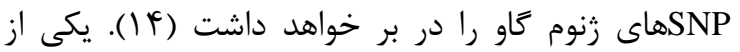

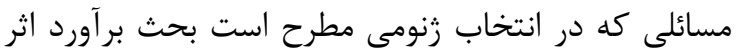

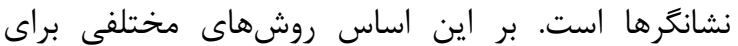

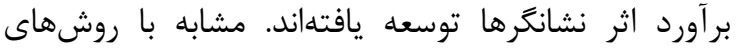

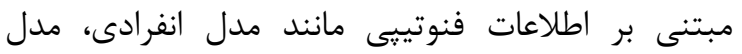

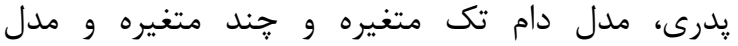

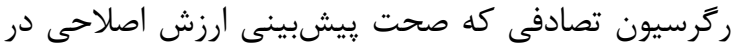

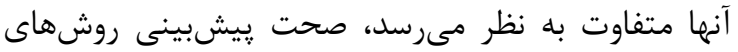

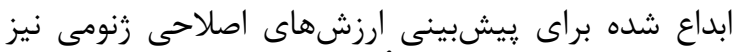

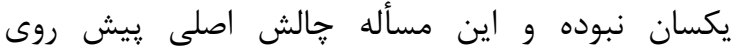

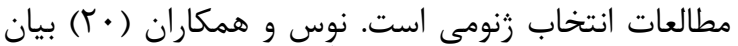

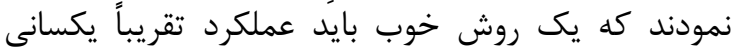

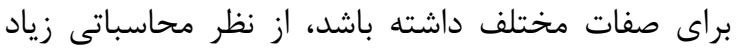

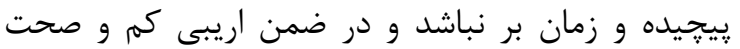
ييشبينى بالا داشته باشد.

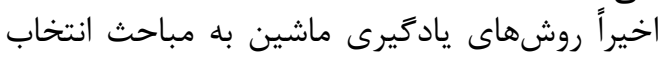

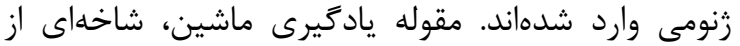

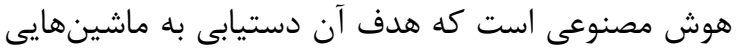

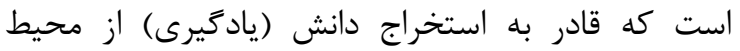

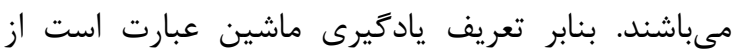

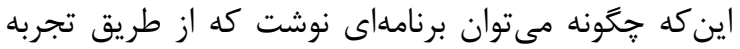

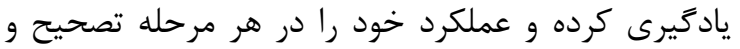

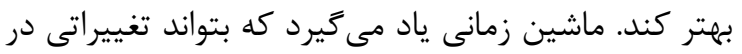

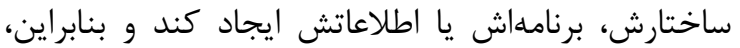

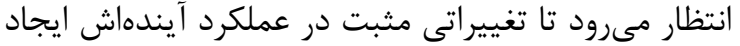

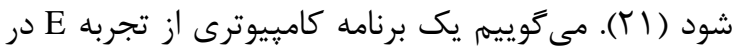

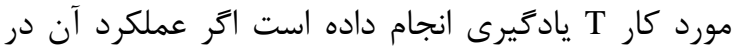

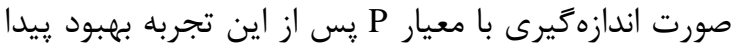

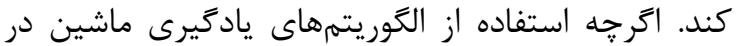

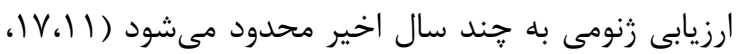

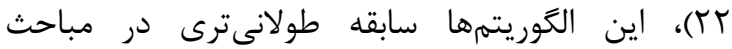

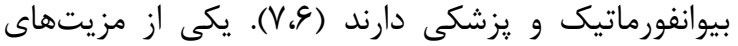

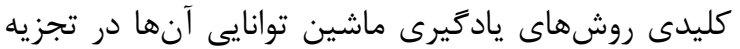

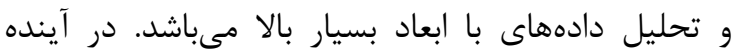

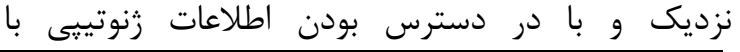




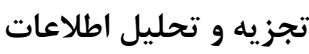

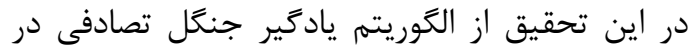

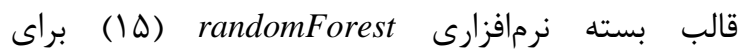

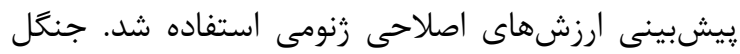

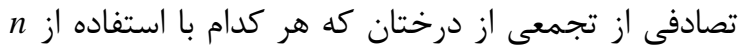

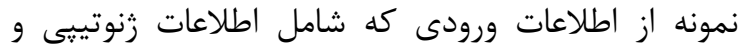

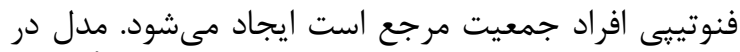

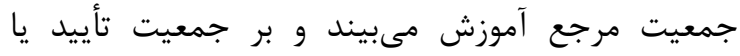

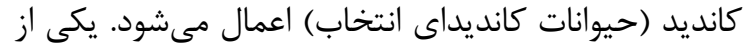

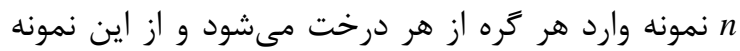

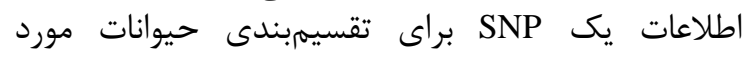

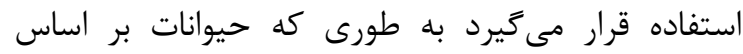

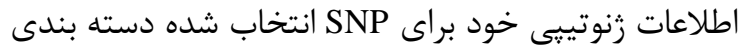

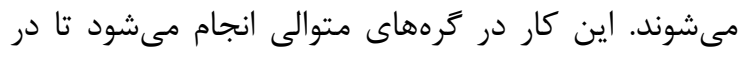

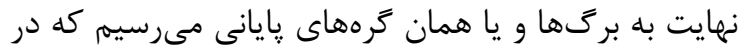

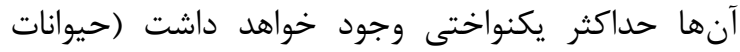

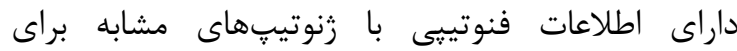

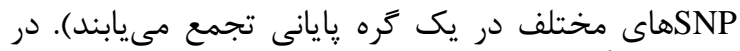

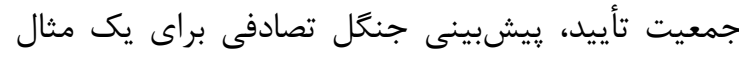

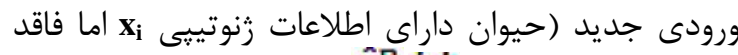

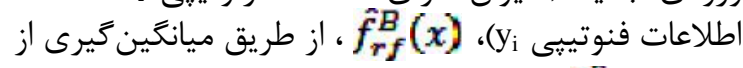
درخت ، د 3 B $f_{r f}^{B}(x)=\frac{1}{B} \sum_{b=1}^{B} T\left(x, \Psi_{b}\right)$,

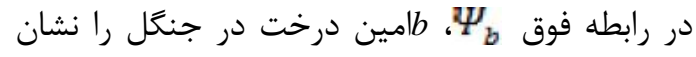

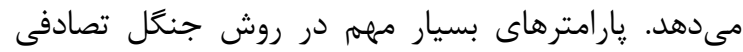

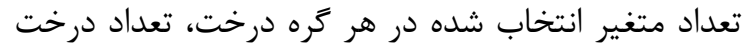

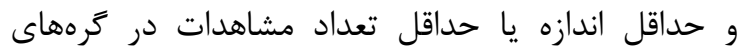

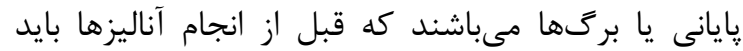

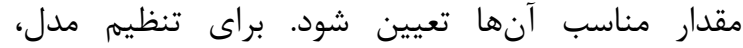

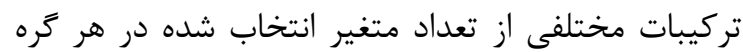

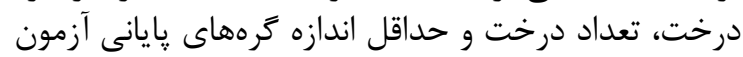

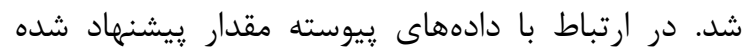

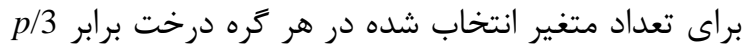

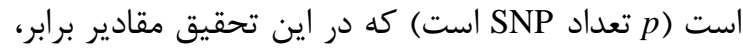

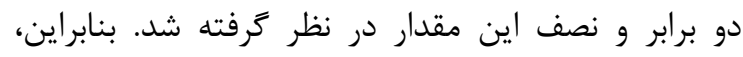

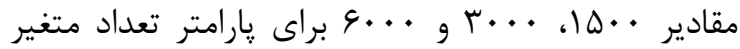

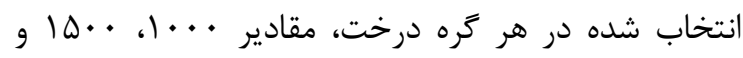

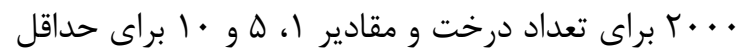

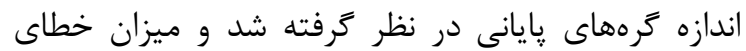
خارج از كيسه براى هر تركيب محاسبه شد (جدائ ندول () ). در

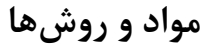 شبيهسازى جمعيت}

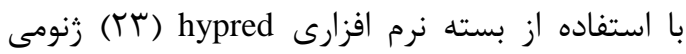

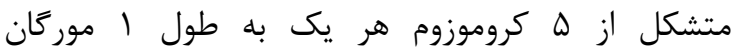

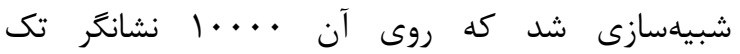

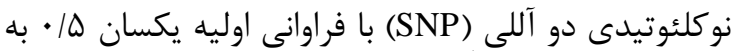

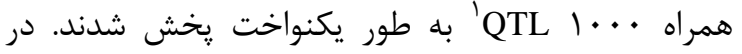

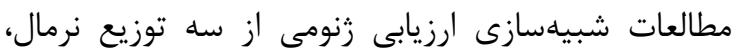

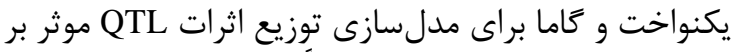

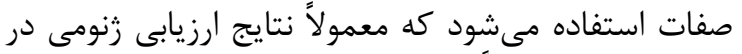

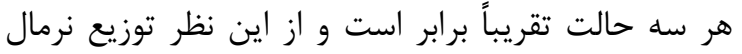

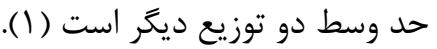

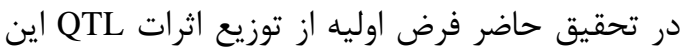

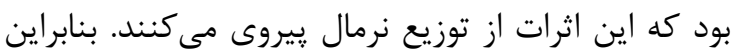

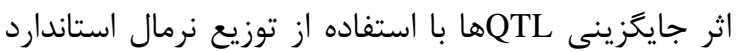

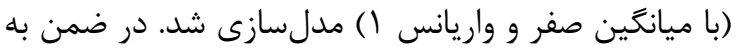

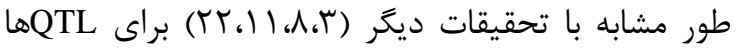

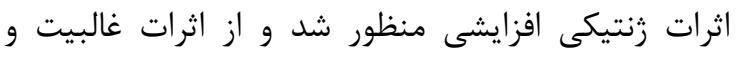

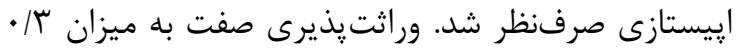

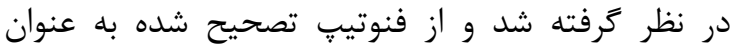

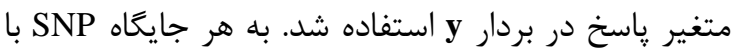

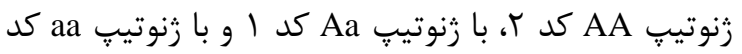

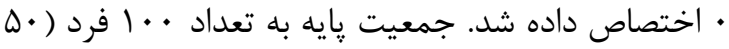

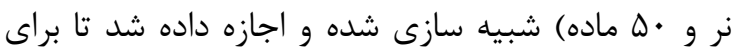

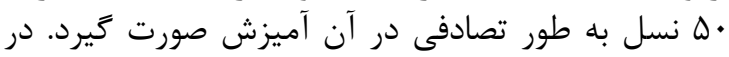

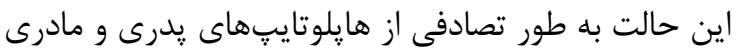

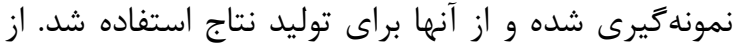

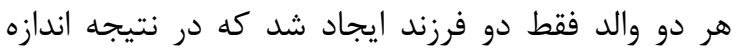

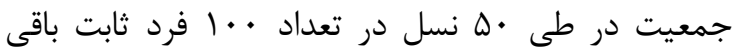

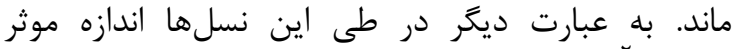

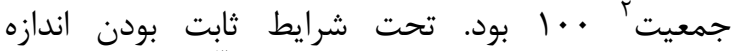

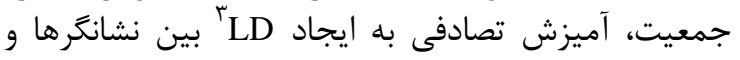

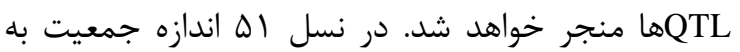

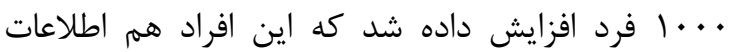

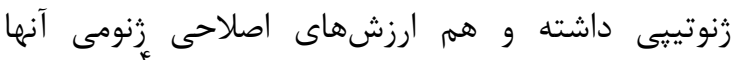

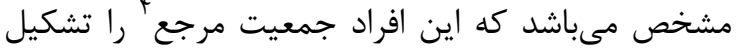

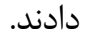

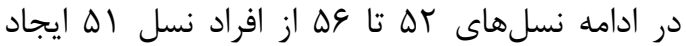

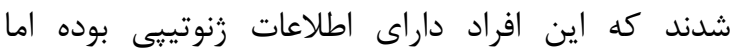

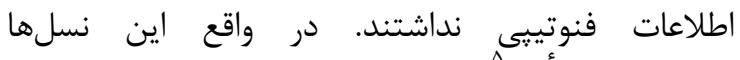

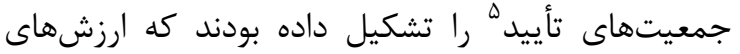

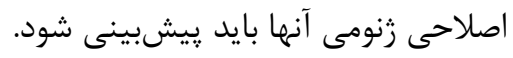

\begin{tabular}{lll}
\hline 1- Quantitative Trait Loci & 2- Effective Population Size $\left(N_{e}\right)$ & 3- Linkage Disequilibrium \\
4- Reference, Training & 5- Test
\end{tabular}




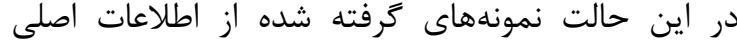

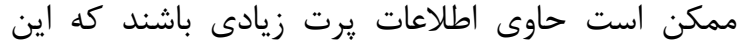

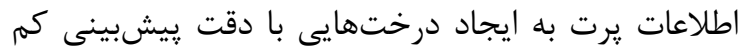

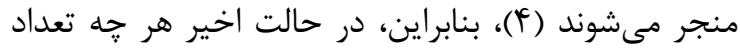

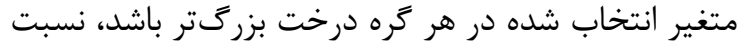

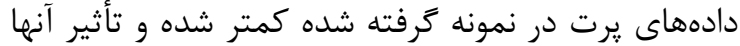

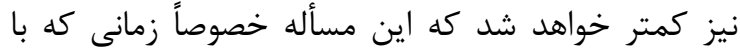

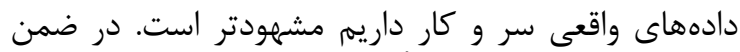

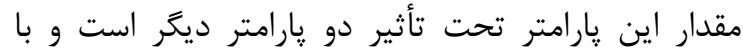

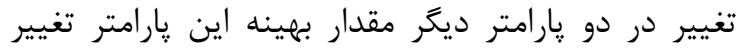

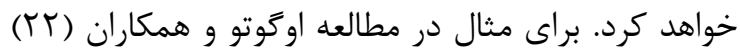

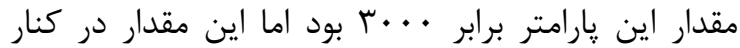

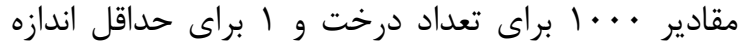

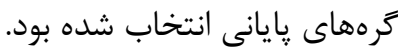

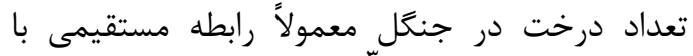

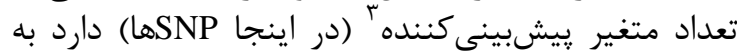

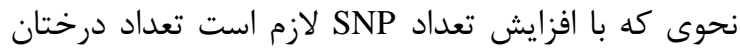

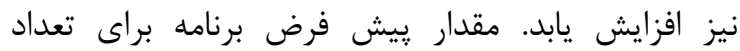

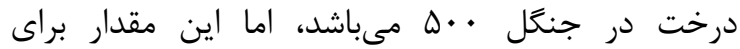
SNP 1.... با افزايش تعداد درخت احتمال اينكه بيشتر SNP نهات

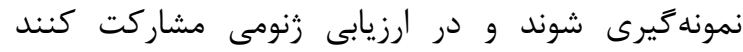

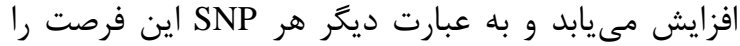

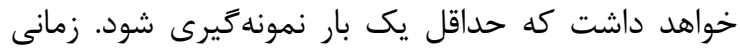

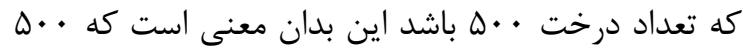

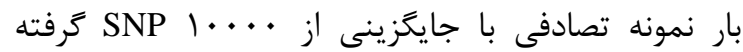

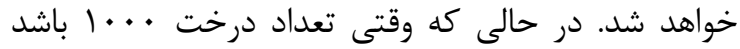

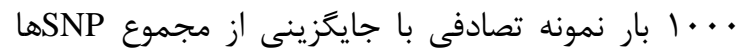

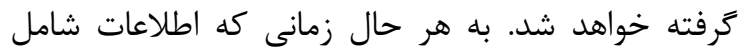

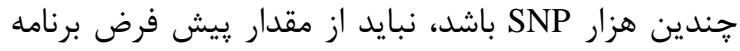

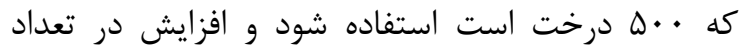

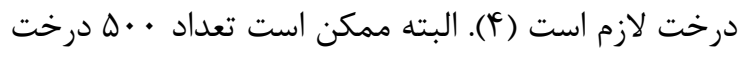

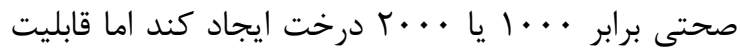
اطمينان اين صحت كم خواهل

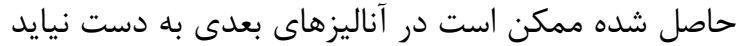

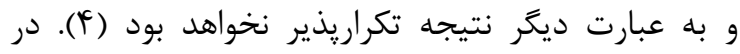

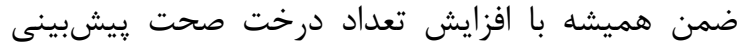

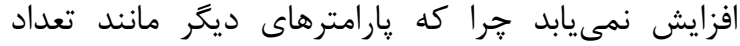

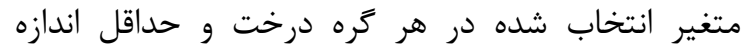

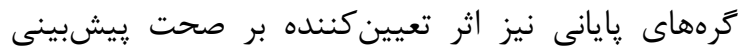

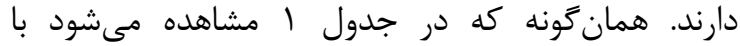

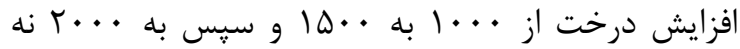
تنها خطا افزايش ييدا كرده بلكه صحت بيش بيشبينى نيز
هر بار نمونهَيرى با جايكزينى از اطلاعات، برخى اطلاعات

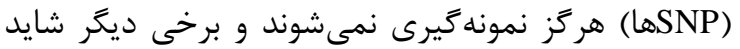

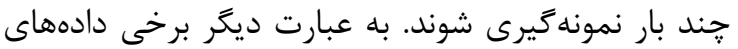

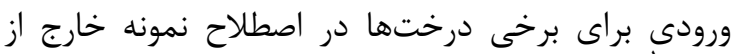

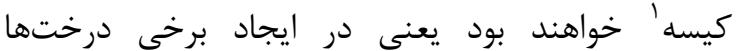
مشاركت نخواهد داشت. اين دادهها عمل ئل يك إند اعتبارسنج

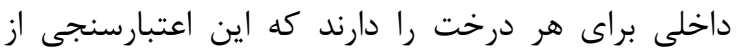

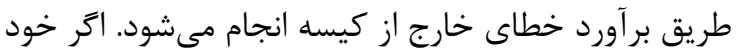

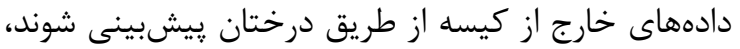

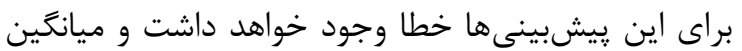

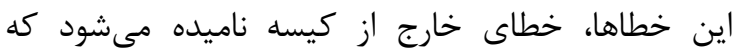

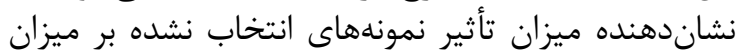

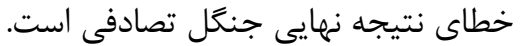

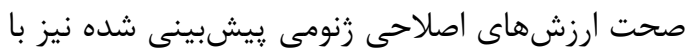

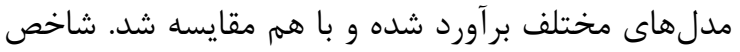

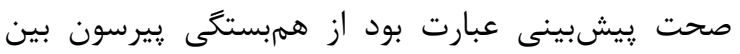

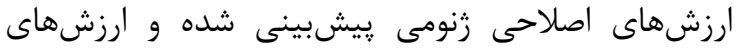
اصلاحى زنومى واقعى (شبيهسازى شدهى).

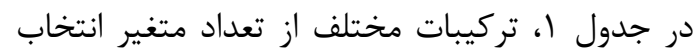

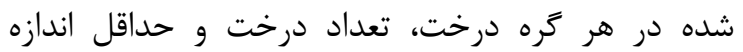

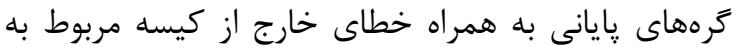

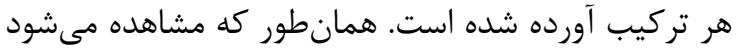

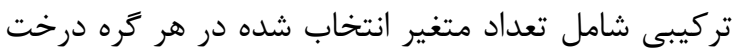

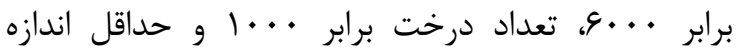

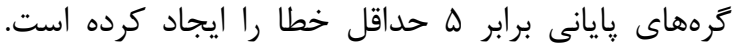

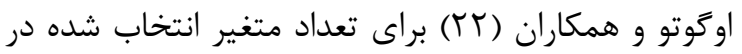

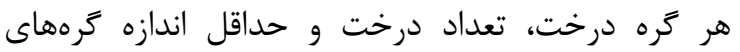

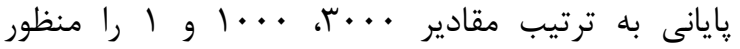

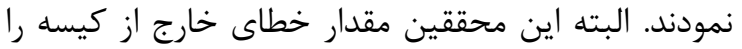

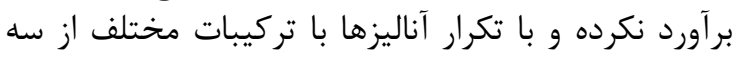

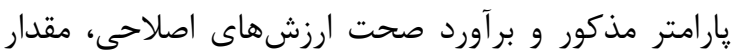

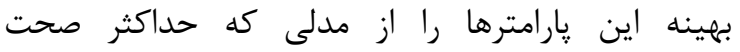

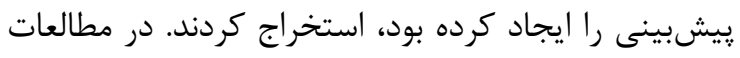

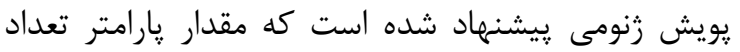

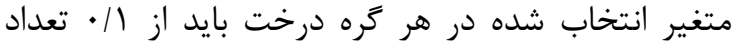

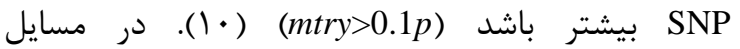

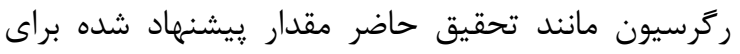

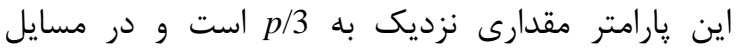

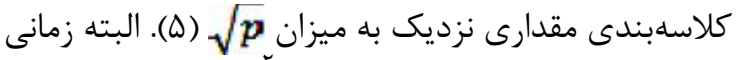

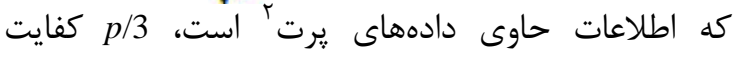

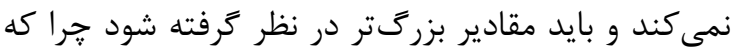


اين مطلب است كه يك رابطه داخلى بين پارامترها برقرار

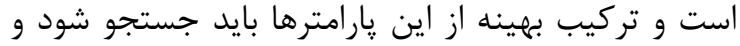
براى تجزيه و تحليل اطلاعات استفاده شود.

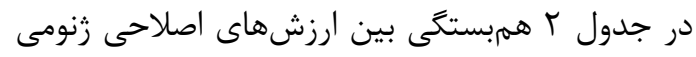

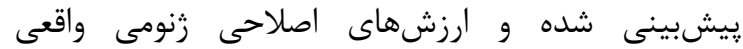

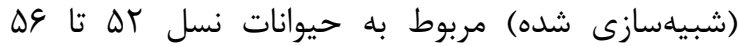

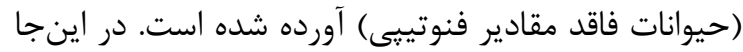

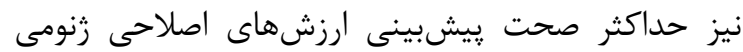

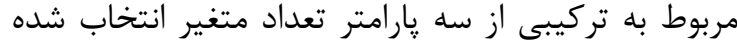

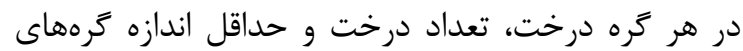

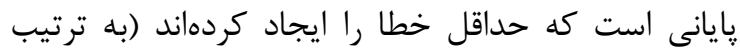

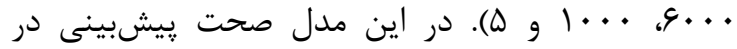
مقايسه با مدلهاى ديكر بالاتر است. نوس و و همكاران

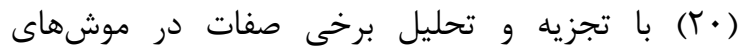

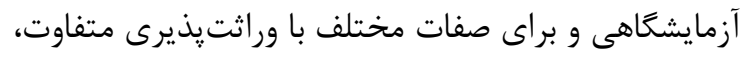

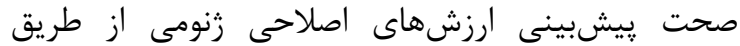

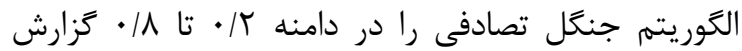

نمودند.
كاهش ييدا كرده است. در اين حالت زمان محاسبات نيز

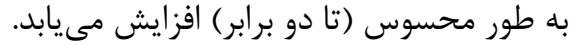

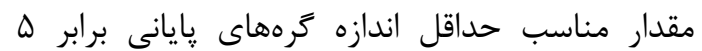

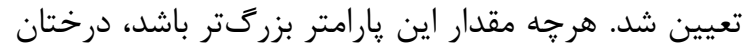

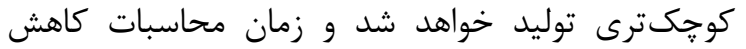

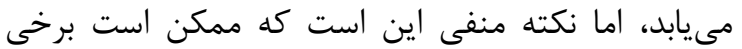

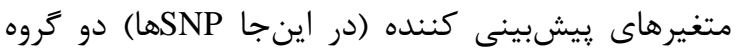

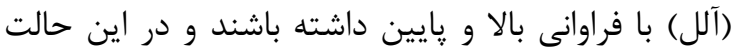

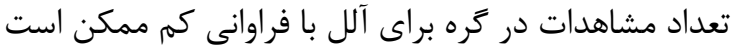

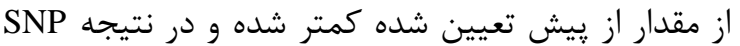

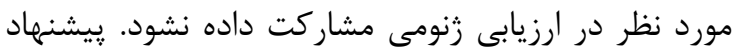

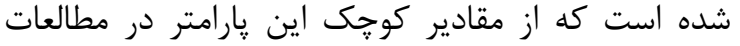

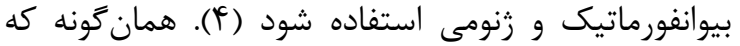

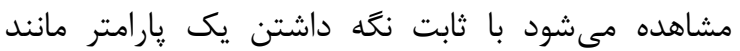

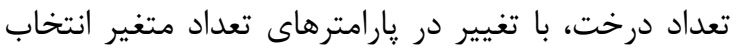

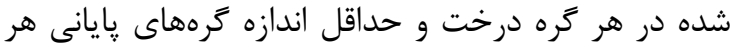

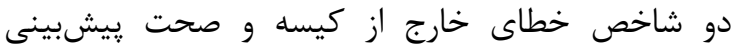
ارزشهاى اصلاحى زنومى تغيير مى خند. نتيجه اخير مؤيد

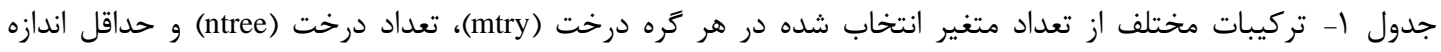

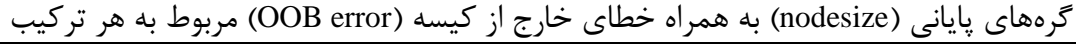

\begin{tabular}{|c|c|c|c|}
\hline ntree & mtry & nodesize & $O O B$ error \\
\hline $1 \cdots$ & $10 \cdots$ & 1 & TFI/TFY \\
\hline $1 \cdots$ & $10 .$. & $\Delta$ & $Y F=|q F|$ \\
\hline $1 \ldots$ & $10 \ldots$ & 1. & TYYIDG. \\
\hline $1 \cdots$ & $r \ldots$ & 1 & rTV/THQ \\
\hline $1 \cdots$ & r... & $\Delta$ & rrG/Far \\
\hline $1 \ldots$ & $r \ldots$ & 1. & r rq/A.. \\
\hline $1 \ldots$ & $4 \ldots$ & 1 & rTD/ATF \\
\hline $1 \ldots$ & $9 \ldots$ & $\Delta$ & rmY/999 \\
\hline $1 \ldots$ & $9 \ldots$ & 1. & $r(T V / T)$. \\
\hline $10 \ldots$ & $10 .$. & 1 & $r r q / 9 \Delta F$ \\
\hline $10 \ldots$ & 10. & $\Delta$ & rrg/lf. \\
\hline $10 \ldots$ & $10 \%$ & 1. & rTV/rAs \\
\hline $10 \ldots$ & $r \ldots$ & 1 & 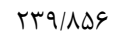 \\
\hline $10 \cdots$ & $r \ldots$ & $\Delta$ & $r$ r \\
\hline $10 \ldots$ & $r \ldots$ & 1. & KTV/DFT \\
\hline $10 \ldots$ & $4 \ldots$ & 1 & $r$ TE/IQF \\
\hline $10 \ldots$ & $4 \ldots$ & $\Delta$ & r TH/GAr \\
\hline $10 \ldots$ & $9 \ldots$ & 1. & $r$ rN/FqT \\
\hline$r \ldots$ & $10 \ldots$ & 1 & $T F \cdot \mid 9 \Delta F$ \\
\hline$r \ldots$ & $10 \ldots$ & $\Delta$ & rrV/VAF \\
\hline$r \ldots$ & $10 .$. & 1. & $r r q / r \Delta F$ \\
\hline$r \ldots$ & $r \ldots$ & 1 & TFT/QTT \\
\hline$r \ldots$ & $r \ldots$ & $\Delta$ & TFI/DF. \\
\hline$r \ldots$ & $r \ldots$ & 1. & TFT/KGI \\
\hline$r \ldots$ & $9 \ldots$ & 1 & TFF/VGD \\
\hline$r \ldots$ & $4 \ldots$ & $\Delta$ & $T F Y / T I Q$ \\
\hline$r \ldots$ & q... & 1. & TFY/GDI \\
\hline
\end{tabular}




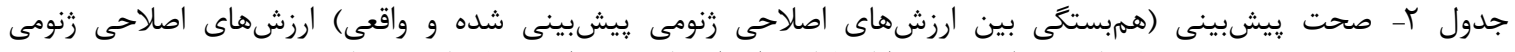

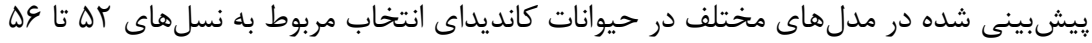

\begin{tabular}{|c|c|c|c|c|c|c|c|}
\hline ntree & mtry & nodesize & G 52 & G 53 & G 54 & G 55 & G 56 \\
\hline $1 \ldots$ & $10 \%$ & $\Delta$ & $\cdot / \Delta V \psi$ & $\cdot / \& \wedge V$ &.$/ 4 T S$ & $\cdot / 4 \cdot V$ & $\cdot / k \cdots$ \\
\hline $1 \ldots$ & $r . .$. & $\Delta$ & $\cdot \mid \Delta \Delta F$ & $\cdot 10 \cdot V$ & . & $\cdot|f| f$ & $\cdot / \pi 9 \Delta$ \\
\hline $1 \ldots$ & q... & $\Delta$ &.$/ 097$ & . & $\cdot / \uparrow \Delta$. & - kfTF & $\cdot|f| \lambda$ \\
\hline $10 \ldots$ & $10 \ldots$ & $\Delta$ & $\cdot \mid D F F$ & . $/ \Delta T K$ & $\cdot|4| 1$ & . IKA & $\cdot / K \wedge \mid$ \\
\hline $10 \ldots$ & r... & $\Delta$ & $\cdot \mid \Delta 99$ & $\cdot|\Delta| \Delta$ & . & $\cdot|4|$. & $\cdot|f| f$ \\
\hline $10 \ldots$ & q... & $\Delta$ & $\cdot \mid \Delta r F$ & $\cdot 10 \cdot 9$ &.$|F| V$ & . & $\cdot /$ rvq \\
\hline$r \ldots$ & $10 .$. & $\Delta$ & $\cdot / \Delta V V$ & $\cdot / \Delta T \Delta$ & ו וא|F. & \& & ו ו \\
\hline r... & r... & $\Delta$ & $\cdot|\Delta r|$ & $\cdot / \Delta T$. & . KTY & . $\mid$ ETI &.$|f| V$ \\
\hline$r \ldots$ & q... & $\Delta$ & $\cdot / \Delta \vee q$ & אוT & - MFTF & אףזן. & •/ КА \\
\hline
\end{tabular}

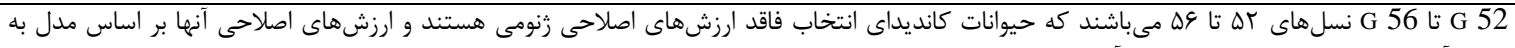
دست آمده از جمعيت مرجع به دست مى مآ مآيد.

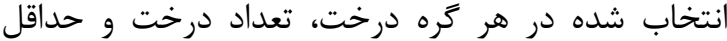

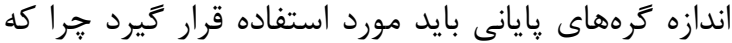

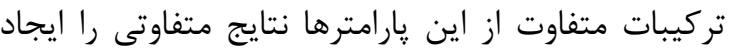

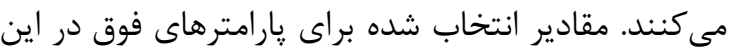

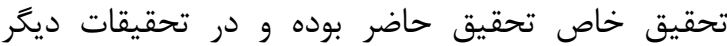

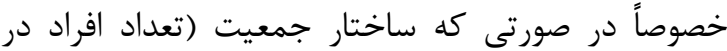

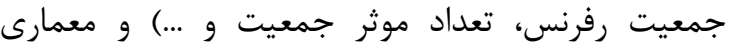

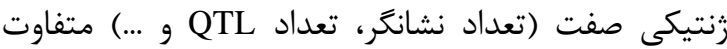

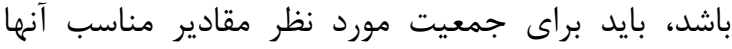

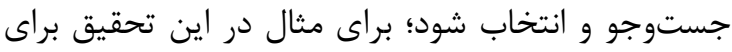

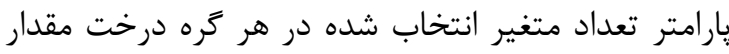

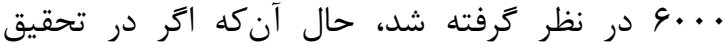

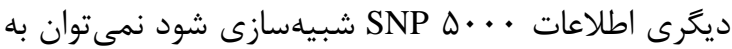

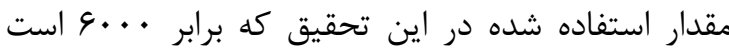

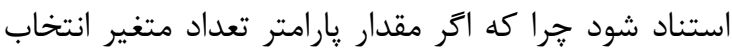

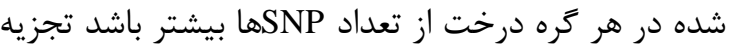

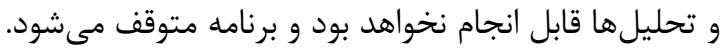

يبشنهادات ارزنده دوست كرانقدر جنان جناب آقاى دكتر

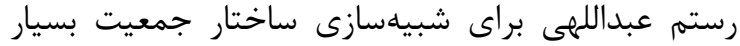
راهخشا بود. بدين وسيله از زحمات ايشان كمال قدردانى

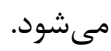

هرجها از جمعيت مرجع دورتر شويم كاهش صحت

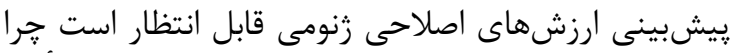

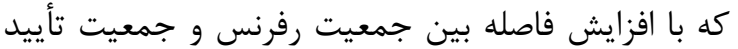

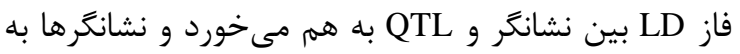

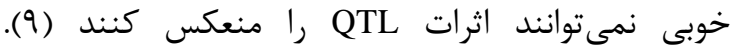

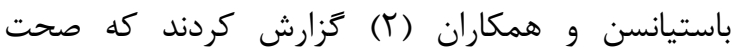

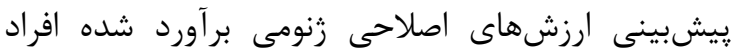

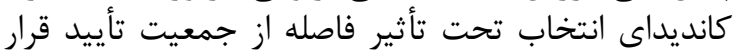

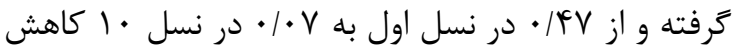

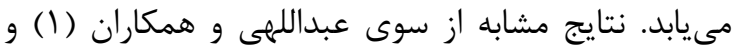

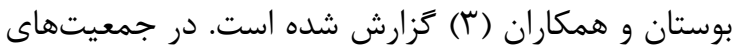

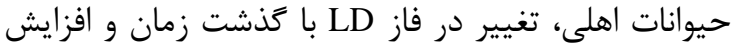

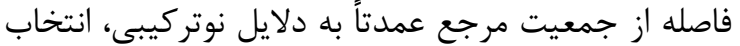

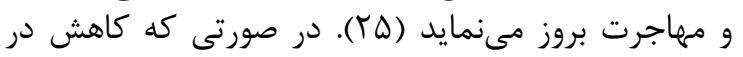

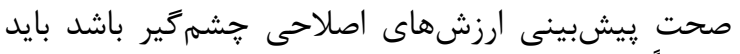

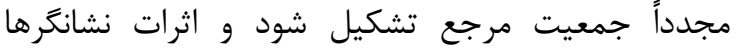

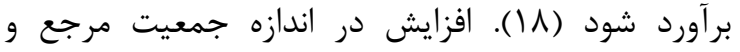

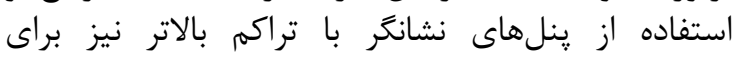

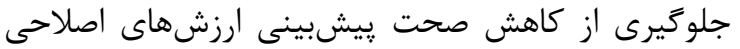

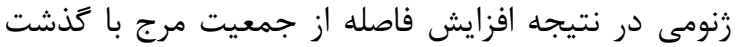

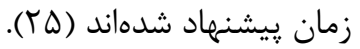

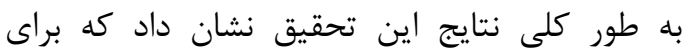

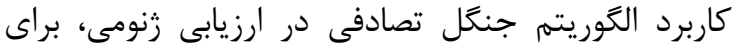

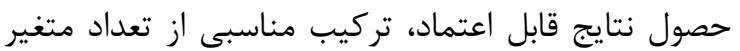



different genomic evaluation methods for traits with different genetic architecture. Animal Production, 15: 65-77.

2. Bastiaansen, J.W.M., A. Coster, M.P.L. Calus, J.A.M. van Arendonk and H. Bovenhuis. 2011. Long-term response to genomic selection: effects of estimation method and reference population structure for different genetic architectures. Genetics Selection Evolution, 44: 3 pp.

3. Boustan, A., A. Nejati-Javaremi, M. Moradi Shahrbabak and M. Saatchi. 2012. Effect of using different number and type of records from different generations as reference population on the accuracy of genomic evaluation. Archiv Tierzucht, 56: $68 \mathrm{pp}$.

4. Boulesteix, A.L., S. Janitza, J. Kruppa and I.R. König. 2012. Overview of random forest methodology and practical guidance with emphasis on computational biology and bioinformatics. Technical Report. Department of Statistics, University of Munich.

5. Breiman, L. 2001. Random forests. Machine Learning, 45: 5-32.

6. Bureau, A., J. Dupuis, B. Hayward, K. Falls and P. Van Eerdewegh. 2003. Mapping complex traits using random forests. BMC Genetics, 4: $64 \mathrm{pp}$.

7. Bureau, A., J. Dupuis, K. Falls, K. Lunetta, B. Hayward and T. Keith. 2005. Identifying SNPs predictive of phenotype using random forests. Genetic Epidemiology, 28: 171-182.

8. Chen, X., M. Wang and H. Zhang. 2011. The use of classification tree for bioinformatics. WIREs Data Mining and Knowledge Discovery, 1: 55-63.

9. Erbe, M., B.J. Hayes, L.K. Matukumalli, S. Goswami, P.J. Bowman, C.M. Reich, B.A. Mason and M.E. Goddard. 2012. Improving accuracy of genomic predictions within and between dairy cattle breeds with imputed high-density single nucleotide polymorphism panels. Journal of Dairy Science, 95: 4114-4129.

10. Goldstein, B.A., A.E. Hubbard, A. Cutler and L.F. Barcellos. 2010. An application of random forests to a genome-wide association dataset: Methodological considerations and new findings. BMC Genetics, 11: 49 pp.

11. González-Recio, O. and S. Forni. 2011. Genome-wide prediction of discrete traits using Bayesian regressions and machine learning. Genetics Selection Evolution, 43: $7 \mathrm{pp}$.

12. Habier, D., R.L. Fernando and J.C. Dekkers. 2007. The impact of genetic relationship information on genome-assisted breeding values. Genetics, 177: 2389-2397.

13. Hill, W.G. 2008. Estimation, effectiveness and opportunities of long term genetic improvement in animals and maize, Lohmann Information, 43: 3-20.

14. Khatkar, M.S., M. Moser, B.J. Hayes and H.W. Raadsma. 2012. Strategies and utility of imputed SNP genotypes for genomic analysis in dairy cattle. BMC Genomics, 13: $538 \mathrm{pp}$.

15. Liaw, A. and M. Wiener. 2002. Classification and regression by random forest. R News. 2:18-22.

16. Meuwissen, T.H.E., B.J. Hayes and M.E. Goddard. 2001. Prediction of total genetic value using genome wide dense marker maps. Genetics, 157: 1819-1829.

17. Moser, G., B. Tier, R.E. Crump, M.S. Khatkar and H.W. Raadsma. 2009. A comparison of five methods to predict genomic breeding values of dairy bulls from genome-wide SNP markers. Genetic Selection Evolution, 41: $56 \mathrm{pp}$.

18. Muir, W.M. 2007. Comparison of genomic and traditional BLUP-estimated breeding value accuracy and selection response under alternative trait and genomic parameters. Journal of Animal Breeding and Genetics, 124: 342-355.

19. Nejati-Javaremi, A., C. Smith and J. Gibson. 1997. Effect of total allelic relationship on accuracy of evaluation and response to selection. Journal of Animal Science, 75: 1738-1745.

20. Neves, H.H.R., R. Carvalheiro and S.A. Queiroz. 2012. A comparison of statistical methods for genomic selection in a mice population. BMC Genetics, 13:100.

21. Nilsson, N.J. 1998. Introduction to Machine Learning. Stanford University. Stanford, USA. 412 pp.

22. Ogutu, J.O., H.P. Piepho and T. Schulz-Streeck. 2011. A comparison of random forests, boosting and support vector machines for genomic selection. BMC Proc. 5(Suppl. 3): S11.

23. Technow, F. 2014. hypred: Simulation of genomic data in applied genetics. Available at: http://cran.rproject.org/web/packages/hypred/hypred.pdf

24. Visscher, P.M. and C.S. Haley. 1998. Power of a chromosomal test to detect genetic variation using genetic markers. Heredity, 81: 317-326.

25. Zhe, Z., Q. Zhang and X.D. Ding. 2011. Advances in genomic selection in domestic animals. Chinese Science Bulletin, 56: 2655-2663. 


\title{
Tuning and Application of Random Forest Algorithm in Genomic Evaluation
}

\author{
Farhad Ghafouri Kesbi ${ }^{1}$, Ghodrat Rahimi Mianji ${ }^{2}$, Mahmoud Honarvar ${ }^{3}$ \\ and Ardeshir Nejati Javaremi ${ }^{4}$

$\begin{array}{r}\hline \hline \text { 1- Ph.D. Student, Sari Agricultural Sciences and Natural Resources University, } \\ \text { (Corresponding Author: email:farhad_ghy@ yahoo.com) } \\ \text { 2- Professor, Sari Agricultural Sciences and Natural Resources University } \\ \text { 3- Assistant Professor, of Animal Science, Islamic Azad University, Shahre Qods Branch, Kara } \\ \text { 4- Assistant Professor, College of Agriculture and Natural Resources, University of Tehran } \\ \text { Received: June 29, } 2014 \quad \text { Accepted: September 21, 2014 } \\ \hline \hline\end{array}$

\begin{abstract}
One of the most important issues in genomic selection is using a decent method for estimating marker effects and genomic evaluation. Recently, machine learning algorithms which are members of non-parametric and non-linear methods have been extended to genomic evaluation. One of these methods is Random Forest (RF) on which this research was focused. Important parameters in RF algorithm are the number of SNPs selected randomly at each tree node (mtry), the number of trees to grow (ntree) and the minimum size of terminal nodes of trees (node size) which need to be predefined before analyses and for them the model should be tuned. A genome comprised of five chromosomes, one Morgan each, on which 10000 bi-allelic SNP were arrayed was simulated and the efficiency of different combinations of mtry, ntree and node size was tested and the best combination was selected based on comparison of accuracy of predicted genomic value as well as OOB error estimates. For the simulated data in the current study the least OOB error as well as the maximum prediction accuracy was related to a model with 6000 mtry, 1000 ntree and 5 node size. Other combinations did not increase the accuracy of prediction while led to an increase in time of analyses for those which used more trees. Since the accuracy of prediction is a function of mtry, ntree and node size, in genomic evaluation, different combinations of these parameters should be used and the combination which caused the maximum prediction accuracy should be used for genomic evaluation.
\end{abstract}

Keywords: Genomic Breeding Value, Genomic Evaluation, Random Forest, Single Nucleotide Marker, Tree 